\title{
Extremal graph packing problems: Ore-type versus Dirac-type
}

\author{
H. A. Kierstead, A. V. Kostochka, and Gexin Yu
}

\begin{abstract}
We discuss recent progress and unsolved problems concerning extremal graph packing, emphasizing connections between Dirac-type and Ore-type problems. Extra attention is paid to coloring, and especially equitable coloring, of graphs.
\end{abstract}

\section{Introduction}

An important instance of combinatorial packing problems is that of graph packing. We say that $n$-vertex graphs $G_{1}, G_{2}, \ldots, G_{k}$ pack, if there exists an edge-disjoint placement of all these graphs onto the same set of $n$ vertices. By definition, two graphs $G_{1}$ and $G_{2}$ pack, if $G_{1}$ is a subgraph of the complement $\bar{G}_{2}$ of $G_{2}$, or, equivalently, $G_{2}$ is a subgraph of the complement $\bar{G}_{1}$ of $G_{1}$. Many basic graph theory problems and concepts can be expressed in a unified (and sometimes more natural) form using the language of graph packing. Here are some relevant examples.

Example 1.1 The problem of existence of a spanning (hamiltonian) cycle in an $n$ vertex graph $G$ (which is a close relative of the famous Travelling Salesman Problem) is equivalent to the question whether the $n$-cycle $C_{n}$ packs with the complement $\bar{G}$ of $G$.

Example 1.2 The independence number $\alpha(G)$ of an $n$-vertex graph $G$ is at least $k$ if and only if $G$ packs with the graph $K_{k}+\bar{K}_{n-k}$ consisting of the $k$-clique and $n-k$ isolated vertices.

Example 1.3 An $n$-vertex graph $G$ is $k$-colorable if and only if $G$ packs with an $n$-vertex graph that is the union of $k$ cliques.

A proper vertex coloring of a graph is equitable if the sizes of its color classes differ by at most one.

Example 1.4 An $n$-vertex graph $G$ is equitably $k$-colorable if and only if $G$ packs with the complement $H(n, k)$ of the Turán Graph $T(n, k)$, i.e. with the $n$-vertex graph whose every component is a complete graph with either $\lfloor n / k\rfloor$ or $\lceil n / k\rceil$ vertices.

Turán-type and Ramsey-type problems also can be naturally stated in the language of graph packing.

Since finding optimal solutions of many graph packing problems is NP-hard, corresponding extremal problems giving sufficient conditions for packing graphs are of great interest. Some well-known theorems of this type can be translated into the language of packing as follows.

Theorem 1.5 (Dirac [24]) If $G$ is an n-vertex graph and $\Delta(G) \leq n / 2-1$, then $G$ packs with the cycle $C_{n}$ of length $n$. 
Theorem 1.6 (Ore [63]) If $G$ is an n-vertex graph and $d(x)+d(y) \leq n-2$ for every edge $x y$ in $G$, then $G$ packs with the cycle $C_{n}$ of length $n$.

Theorem 1.7 (Hajnal and Szemerédi [31]) Let $G$ be an n-vertex graph with $\Delta(G) \leq r$. Then $G$ packs with the graph $H(n, r+1)$, whose components are complete graphs with either $\lfloor n /(r+1)\rfloor$ or $\lceil n /(r+1)\rceil$ vertices.

Ore's theorem motivates considering the notion of Ore-degree, $\theta(x y)$, of an edge $x y$ in a graph $G$ as the sum, $d(x)+d(y)$, of the degrees of its ends in $G$. By definition, the Ore-degree of an edge $x y$ is two greater than the degree of the vertex $x y$ in the line graph of $G$, and coincides with the degree of $x y$ in the total graph of $G$. We let the Ore-degree of a graph $G$ be $\theta(G)=\max _{x y \in E(G)} \theta(x y)$. Thus, Ore's theorem says that every $n$-vertex graph $G$ with $n \geq 3$ and $\theta(G) \leq n-2$ packs with the cycle $C_{n}$. Observe that $\theta(G)$ is closely related to the maximum degree of the line graph $L(G)$ :

$$
\theta(G)=\Delta(L(G))+2
$$

and that any bound on $\theta(G)$ is in fact a bound on $\Delta(L(G))$. Observe also that for every graph $G$,

$$
\Delta(G)+\delta^{*}(G) \leq \theta(G) \leq 2 \Delta(G),
$$

where $\delta^{*}(G)$ is the minimum positive degree of a vertex in $G$. The left inequality in (1.1) is obtained by considering an edge $x y \in E(G)$ incident with a vertex of the maximum degree.

In view of Dirac's and Ore's theorems, we call upper bounds in terms of maximum degree giving sufficient conditions for packing graphs Dirac-type bounds and those in terms of the Ore-degree Ore-type bounds.

In this survey, we compare recent progress in Dirac-type and Ore-type bounds for graph packing problems. We discuss the general problem in the next section, packing a graph with a power of a cycle or path in Section 3, graph coloring in Section 4, equitable coloring in Section 5, and equitable list coloring in Section 6.

We use the standard notation. In particular, for a graph $G,|G|$ denotes the order and $\|G\|$ denotes the size of $G$.

\section{General packing results}

Some milestone results on extremal graph packing problems were obtained in the seventies. In the same issue of the Journal of Combinatorial Theory (B), fundamental papers by Bollobás and Eldridge [10] and Sauer and Spencer [68] appeared. The papers gave sufficient conditions for packing of two graphs with given average or maximum degree. Some of these results were also obtained by Catlin in his Ph.D. Thesis [16] and in [15]. In particular, Sauer and Spencer [68] proved the following Dirac-type bound.

Theorem 2.1 (Sauer and Spencer [68]) If $G_{1}$ and $G_{2}$ are n-vertex graphs and $2 \Delta\left(G_{1}\right) \Delta\left(G_{2}\right)<n$, then $G_{1}$ and $G_{2}$ pack.

The proof is simple (we will show it for a half-Ore version), but the bound is sharp for even $n$. 
Example 2.2 Let $G_{1}$ be a perfect matching on $n$ vertices, and $G_{2}$ contain $K_{1+n / 2}$ and have maximum degree $n / 2$. Then $2 \Delta\left(G_{1}\right) \Delta\left(G_{2}\right)=2 \cdot 1 \cdot n / 2$ but $G_{1}$ and $G_{2}$ do not pack, since in any packing of $G_{1}$ and $G_{2}$ only one end of each edge of $G_{1}$ can be placed onto a vertex in the copy of $K_{1+n / 2}$ in $G_{2}$.

Example 2.3 Let $n \equiv 2(\bmod 4)$. Let $G_{1}$ be a perfect matching on $n$ vertices, and $G_{2}=K_{n / 2, n / 2}$. Again $2 \Delta\left(G_{1}\right) \Delta\left(G_{2}\right)=2 \cdot 1 \cdot n / 2$. Again, $G_{1}$ and $G_{2}$ do not pack, since in any packing of $G_{1}$ and $G_{2}$, the ends of each edge of $G_{1}$ should be placed into the same partite set of $K_{n / 2, n / 2}$, but $n / 2$ is odd.

Kaul and Kostochka [35] proved that Examples 2.2 and 2.3 are the only examples where the bound of Sauer and Spencer is attained.

The half-Ore version of Theorem 2.1 mentioned above is as follows.

Theorem 2.4 If $G_{1}$ and $G_{2}$ are $n$-vertex graphs and

$$
\theta\left(G_{1}\right) \Delta\left(G_{2}\right)<n
$$

then $G_{1}$ and $G_{2}$ pack.

Proof. For $i \in[2]$, set $G_{i}=\left(V_{i}, E_{i}\right), d_{i}(v)=d_{G_{i}}(v)$, and $\Delta_{i}=\Delta\left(G_{i}\right)$. A packing of $G_{2}$ with $G_{1}$ will be viewed as a mapping $f$ of $V_{1}$ onto $V_{2}$ such that if $u v \in E_{1}$ then $f(u) f(v) \notin E_{2}$. We argue by induction on $\left\|G_{1}\right\|$. The base step $\left\|G_{1}\right\|=0$ is trivial, so consider the induction step. Let $x$ be a vertex with the minimum positive degree $\delta^{*}$ in $G_{1}$ and $x y$ be any edge incident with $x$. By the induction hypothesis there exists a packing $f$ of $G_{1}-x y$ with $G_{2}$. If $f(x) f(y) \notin E_{2}$, then we are done. Otherwise, we will show that there is some good $z \in V-y$ such that the mapping $g_{z}$ obtained from $f$ by switching the images of $x$ and $z$ is a packing of $G_{1}$ with $G_{2}$. Suppose $z \in V-y$ is bad, i.e., not good. Then there exists an edge $u v \in E_{1}$ with $g_{z}(u) g_{z}(v) \in E_{2}$. Since $f$ packs $G_{1}-x y$ with $G_{2}$, at least one, say $v$, of $u, v$ is in $\{x, z\}$. Then either (1) $x u \in E_{1}$ and $g_{z}(x) g_{z}(u)=f(z) f(u) \in E_{2}$ or $(2) z u \in E_{1}$ and $g_{z}(z) g_{z}(u)=f(x) f(u) \in E_{2}$. The number of $z$ for which (1) holds is at most $d_{1}(x) \Delta_{2}$ : There are at most $d_{1}(x)$ choices for $u$ and each choice witnesses at most $\Delta_{2}$ different $z$. Similarly, the number of $z$ for which (2) holds is at most $d_{2}(f(x)) \Delta_{1}$. Noting that the choice $z=x, u=y$ has been counted twice, and using both (1.1) and (2.1), the total number of such bad $z$ is at most

$$
d_{1}(x) \Delta_{2}+d_{2}(f(x)) \Delta_{1}-1 \leq\left(\delta^{*}+\Delta_{1}\right) \Delta_{2}-1 \leq \theta\left(G_{1}\right) \Delta_{2}-1<n-1=\left|V_{1}-y\right| .
$$

Thus there exists a good $z \in V_{1}-y$, and so $g_{z}$ is a packing of $G_{1}$ with $G_{2}$.

A sharpening of this result was proved in [55]:

Theorem 2.5 If two n-vertex graphs $G_{1}$ and $G_{2}$ satisfy $\theta\left(G_{1}\right) \Delta\left(G_{2}\right) \leq n$, then $G_{1}$ and $G_{2}$ pack, with the following exceptions:

1. $G_{1}$ is a perfect matching and $G_{2}$ either is $K_{n / 2, n / 2}$ with $n / 2$ odd or contains $K_{n / 2+1}$;

2. $G_{2}$ is a perfect matching, and $G_{1}$ either is $K_{r, n-r}$ with $r$ odd or contains $K_{n / 2+1}$. 
Theorem 2.5 shows that Theorem 2.4 has extremal graphs other than Examples 2.2 and 2.3, but not many. Observe also that the conditions in Theorems 2.5 and 2.4 involve $\Delta\left(G_{2}\right)$. The following Ore-type analogue of Theorem 2.1 was conjectured in $[56]$.

Conjecture 2.6 If $G_{1}$ and $G_{2}$ are n-vertex graphs and $\theta\left(G_{1}\right) \theta\left(G_{2}\right)<2 n$, then $G_{1}$ and $G_{2}$ pack.

The conjecture looks natural and maybe has a simple proof, but so far we have failed to find any proof.

The main conjecture in the area (strengthening Theorem 2.1) is the following BEC-conjecture:

Conjecture 2.7 If $G_{1}$ and $G_{2}$ are $n$-vertex graphs with maximum degrees $\Delta_{1}$ and $\Delta_{2}$, respectively, and $\left(\Delta_{1}+1\right)\left(\Delta_{2}+1\right) \leq n+1$, then $G_{1}$ and $G_{2}$ pack.

This conjecture was posed by Bollobás and Eldridge [9] (see also [8, 10]) and independently by Catlin [16].

The following examples show that the conjecture is sharp for all values of $\Delta\left(G_{1}\right)$ and $\Delta\left(G_{2}\right)$, if true.

Example 2.8 Let positive integers $\Delta_{1}$ and $\Delta_{2}$ be fixed. Let $G_{1}$ be the disjoint union of $\Delta_{2}$ copies of $K_{\Delta_{1}+1}$ and one copy of $K_{\Delta_{1}-1}$. Let $G_{2}$ be the disjoint union of $\Delta_{1}$ copies of $K_{\Delta_{2}+1}$ and one copy of $K_{\Delta_{2}-1}$. Then $\left|V\left(G_{1}\right)\right|=\left|V\left(G_{2}\right)\right|=\left(\Delta_{1}+\right.$ 1) $\left(\Delta_{2}+1\right)-2$. Suppose that $G_{1}$ and $G_{2}$ pack. Then in this packing, each of the $\Delta_{1}$ copies of $K_{\Delta_{2}+1}$ in $G_{2}$ should intersect all the $\Delta_{2}+1$ components of $G_{1}$. But the $K_{\Delta_{1}-1}$-component of $G_{1}$ cannot meet all these $\Delta_{1}$ copies of $K_{\Delta_{2}+1}$.

Example 2.9 Let a positive integer $\Delta_{1}$ and a positive odd integer $\Delta_{2}$ be fixed. Let $G_{1}$ be the disjoint union of $\Delta_{2}$ copies of $K_{\Delta_{1}+1}$ and one copy of $K_{\Delta_{1}-1}$. Let $G_{2}$ be the disjoint union of $\Delta_{1}-1$ copies of $K_{\Delta_{2}+1}$ and one copy of $K_{\Delta_{2}, \Delta_{2}}$. Again $\left|V\left(G_{1}\right)\right|=\left|V\left(G_{2}\right)\right|=\left(\Delta_{1}+1\right)\left(\Delta_{2}+1\right)-2$. Suppose that $G_{1}$ and $G_{2}$ pack. In this packing, each of the $\Delta_{1}-1$ copies of $K_{\Delta_{2}+1}$ in $G_{2}$ should intersect all the $\Delta_{2}+1$ components of $G_{1}$. This leaves two vertices in each clique of size $\Delta_{1}+1$ in $G_{1}$ and $K_{\Delta_{2}, \Delta_{2}}$ in $G_{2}$. So we come to Example 2.3 of graphs that do not pack.

Only very special cases of the BEC-conjecture have been proved. In particular, the Hajnal-Szemerédi Theorem (Theorem 1.7) on equitable colorings verifies the conjecture in the case when $G_{2}$ is the disjoint union of cliques of the same size. Aigner and Brandt [1] and independently (for huge $n$ ) Alon and Fisher [2] settled the conjecture in the case $\Delta_{1} \leq 2$ (this particular case was conjectured by Sauer and Spencer [68]). Csaba, Shokoufandeh, and Szemerédi [21] proved the BECconjecture for $\Delta_{1}=3$ and huge $n$. Bollobás, Kostochka and Nakprasit [12] showed that although the BEC-conjecture is sharp, if one of the two graphs is sparse, to be precise, $d$-degenerate for a small $d$, then much weaker conditions on $\Delta_{1}$ and $\Delta_{2}$ imply the existence of a packing. Recall that a graph $G$ is $d$-degenerate if every subgraph $G^{\prime}$ of $G$ has a vertex of degree (in $G^{\prime}$ ) at most $d$. In this case, the vertices of $G$ can be ordered so that each vertex has fewer than $\operatorname{col}(G):=d+1$ neighbors that precede it. 
Theorem 2.10 (Bollobás, Kostochka and Nakprasit [12]) Let $d \geq 2$. Let $G_{1}$ be a d-degenerate graph of order $n$ and maximum degree $\Delta_{1}$ and $G_{2}$ be a graph of order $n$ and maximum degree at most $\Delta_{2}$. If

$$
\begin{gathered}
40 \Delta_{1} \ln \Delta_{2}<n \quad \text { and } \\
40 d \Delta_{2}<n,
\end{gathered}
$$

then there is a packing of $G_{1}$ and $G_{2}$.

If $\Delta_{2} \geq 215$, then $\Delta_{2} / \ln \Delta_{2} \geq 40$. Therefore, Theorem 2.10 yields that the BECconjecture holds if $\Delta_{2} \geq 215$ and $\Delta_{1} \geq 40 \mathrm{~d}$. Adapting the proof of Theorem 2.10 to control the maximum degree of the union of the two packed graphs, implies the following result on simultaneous packing of many graphs.

Theorem 2.11 (Bollobás, Kostochka and Nakprasit [12]) Let $n, d, \Delta$ and $q$ be positive integers such that $d \geq 2, q \leq \frac{n}{1500 d^{2}}$, and $1000 d \Delta<\frac{n}{\ln n}$. Let $F_{1}, \ldots, F_{q}$ be $d$-degenerate graphs of order $n$ and maximum degree at most $\Delta$. Then $F_{1}, \ldots, F_{q}$ pack.

For a fixed $d$, Theorem 2.11 allows packing linearly many (in $n$ ) $d$-degenerate $n$-vertex graphs of moderate maximum degree. The phenomenon here is that it is easier to pack graphs if the number of vertices is significantly greater than the maximum degrees of the graphs to be packed.

Clearly, restriction (2.3) cannot be weakened by more than 40 times. The following result in [11] shows that (2.2) is also weakest up to a constant factor.

Theorem 2.12 (Bollobás, Kostochka and Nakprasit [11]) Let $k$ be a positive integer and $q$ be a prime power. Then for every $n \geq q \frac{q^{k+1}-1}{q-1}$, there are graphs $G_{1}(n, k)$ and $G_{2}(n, q, k)$ of order $n$ that do not pack and have the following properties.

(a) $G_{1}(n, k)$ is a forest with $n-k$ edges and maximum degree at most $n / k$;

(b) $G_{2}(n, q, k)$ is a $\frac{q^{k}-1}{q-1}$-degenerate graph with maximum degree at most $2 n / q$.

So, for $l \geq 5, q=3^{l}, k \geq l$, and $n=\frac{3}{2}\left(3^{k+1}-1\right)$, consider the graphs $G_{1}=$ $G_{1}(n, k)$ and $G_{2}=G_{2}\left(n, 3^{l}, k\right)$ of Theorem 2.12. Then (2.3) holds for $l>4$ (we need $d \geq 2$ in the theorem), so the bounding inequality is (2.2). Furthermore, $\Delta\left(G_{1}\right) \ln \Delta\left(G_{2}\right) \leq \frac{n}{k} \ln n<\frac{n}{k}(1+(k+1) \ln 3)<2 n$, so restriction $(2.2)$ can be possibly weakened only by a constant factor.

Motivated by Theorem 2.10, Theorem 2.1 has very recently been strengthened [44] in terms of game coloring number. This parameter is defined by means of the marking game, which is played by two players Alice and Bob on a graph G. Bob decides who plays first, and then the players take turns choosing unchosen vertices until all vertices have been chosen. Let $L$ be the order in which the vertices are chosen. The score of the game is the least integer $s$ such that every vertex has fewer than $s$ neighbors that precede it in $L$. The game coloring number $\operatorname{gcol}(G)$ is the least integer $k$ such that Alice has a strategy for obtaining a score of at most $k$ regardless of how Bob plays. The new result says: 
Theorem 2.13 ([44]) If two graphs $G_{1}$ and $G_{2}$ satisfy

$$
\left(\operatorname{gcol}\left(G_{1}\right)-1\right) \Delta\left(G_{2}\right)+\left(\operatorname{gcol}\left(G_{2}\right)-1\right) \Delta\left(G_{1}\right)<n,
$$

then they pack.

Theorem 2.13 strengthens Theorem 2.1, since for every graph $G$,

$$
\operatorname{col}(G) \leq \operatorname{gcol}(G) \leq \Delta+1 .
$$

Moreover, for some important classes of graphs the upper bound on $\operatorname{gcol}(G)$ can be greatly improved. For instance, Zhu [81] showed that every planar graph $G$ satisfies $\operatorname{gcol}(G) \leq 17$.

In [36], the BEC-conjecture was attacked from a different direction: instead of proving the conjecture for another class of graphs, the following weaker bound for all graphs with high maximum degrees was proved.

Theorem 2.14 ([36]) Let $G_{1}$ and $G_{2}$ be n-vertex graphs with maximum degrees $\Delta_{1}$ and $\Delta_{2}$, respectively. If $\Delta_{1}, \Delta_{2} \geq 300$ and $\left(\Delta_{1}+1\right)\left(\Delta_{2}+1\right) \leq 0.6 n+1$, then $G_{1}$ and $G_{2}$ pack.

This improves the bound of the Sauer-Spencer Theorem for large $\Delta_{1}$ and $\Delta_{2}$ and thus partially answers Problem 4.4 in [32]. Helpful concepts used in the proof are those of critical pairs and of cyclic switchings. Cyclic switchings generalize the ordinary switchings used in the proofs of Theorem 2.4 and the original Sauer-Spencer Theorem.

In all extremal examples $\left(G_{1}, G_{2}\right)$ for the BEC-conjecture that we know, $G_{1}$ contains $\Delta\left(G_{1}\right)$-regular and $G_{2}$ contains $\Delta\left(G_{2}\right)$-regular components. This fact and Theorem 2.10 suggest that maybe the following Ore-type analogue of the BECconjecture holds.

Conjecture 2.15 ([56]) If $G_{1}$ and $G_{2}$ are $n$-vertex graphs and

$$
\left(0.5 \theta\left(G_{1}\right)+1\right)\left(\Delta\left(G_{2}\right)+1\right) \leq n+1,
$$

then $G_{1}$ and $G_{2}$ pack.

The case of Conjecture 2.15 when $G_{2}$ is the union of vertex disjoint triangles (which is the analogue of Corrádi-Hajnal Theorem [18]) was proved independently by Enomoto [26] and Wang [75]. The following extension of the results of Enomoto and Wang is a small step towards Conjecture 2.15.

Theorem 2.16 ([57]) Each $n$-vertex graph $G$ with $\theta(G) \leq \frac{4 n-8}{3}$ packs with any $n$-vertex graph $H$ such that each component of $H$ is either $C_{3}$, or $K_{2}$, or $C_{5}$, or $K_{4}-e$, or a vertex.

Yet another way to attack the BEC-conjecture gives the concept of near packing introduced by Eaton [25]. This is an extension of the notion of defective coloring. In a near packing of degree $d$, the copies of the two graphs may overlap so that the maximum degree of the subgraph spanned by the edges common to both copies is at most $d$. Thus a near packing of degree 0 is an ordinary packing. The following result was proved in [25]: 
Theorem 2.17 (Eaton [25]) Let $0<a \leq 1$. If $G_{1}$ and $G_{2}$ are n-vertex graphs, $\Delta\left(G_{1}\right)+\Delta\left(G_{2}\right) \leq n+a-1$, and $\Delta\left(G_{1}\right) \cdot \Delta\left(G_{2}\right)<$ an, then there exists a near packing of $G_{1}$ and $G_{2}$ of degree $d$ for some integer $d<2 a$. Furthermore, if $\left(\Delta\left(G_{1}\right)+\right.$ $1)\left(\Delta\left(G_{2}\right)+1\right) \leq n+1$, then there exists a near packing of $G_{1}$ and $G_{2}$ of degree 1 .

Eaton also posed the conjecture below and showed that it is sharp if true:

Conjecture 2.18 (Eaton [25]) Let $G_{1}$ and $G_{2}$ be n-vertex graphs and $p$ be a positive real number. If

$$
n \geq\left\lfloor\frac{\Delta\left(G_{1}\right)}{p}\right\rfloor\left(\Delta\left(G_{2}\right)+1\right)+\left\lfloor\frac{\Delta\left(G_{2}\right)}{p}\right\rfloor\left(\Delta\left(G_{1}\right)+1-p\left\lfloor\frac{\Delta\left(G_{1}\right)}{p}\right\rfloor\right),
$$

then there exists a near packing of $G_{1}$ and $G_{2}$ of degree less than $p$.

It would be interesting to prove the Ore-version or half-Ore version of Theorem 2.17. For example, is it true that if $\left(0.5 \theta\left(G_{1}\right)+1\right)\left(\Delta\left(G_{2}\right)+1\right) \leq n+1$, then there exists a near packing of $G_{1}$ and $G_{2}$ of degree 1 ?

Other interesting results on graph packing were obtained by Brandt [14], Csaba [19, 20], Fan and Kierstead [28, 29], Komlós [47], Komlós, Sárkőzy and Szemerédi [49, 50, 51], Sauer and Wang [69], Wozniak [37, 67], Yap [72, 73, 78] and others. One can look into the 75-page survey [77] of the topic by Wozniak. Some of these results are discussed in the next section. After this paper was accepted, we learned about the following very interesting result by Kühn, Osthus and Treglown [59]: for every fixed graph $H$ and large $n$, they found asymptotically exact Ore-type conditions for an $n$-vertex graph $G$ of order divisible by $|H|$ to contain a perfect $H$-packing of $G$, i.e., to contain vertex-disjoint copies of $H$ that cover $V(G)$.

\section{Packing a graph with a power of a cycle}

Let $H=v_{1} v_{2} \cdots v_{n}$ be a path or a cycle. An $r$-chord is an edge of the form $v_{i} v_{i+r}$, where addition is modulo $n$ in the case that $H$ is a cycle. The $r$-th power of $H$ is the graph obtained by adding all $i$-chords with $i \leq r$. The second power of $H$ is called the square of $H$. Pósa (see [27]) conjectured that every graph on $n$ vertices with minimum degree at least $\frac{2}{3} n$ contains the square of $C_{n}$. Seymour [70] strengthened Pósa's Conjecture as follows.

Conjecture 3.1 (Seymour [70]) Every graph $G$ on $n$ vertices with $\delta(G) \geq \frac{r}{r+1} n$ contains the $r$-th power of $C_{n}$.

The case $r=1$ is Dirac's Theorem and the case $r=2$ is Pósa's Conjecture. Rephrased in terms of packing, Seymour's Conjecture looks like this.

Conjecture 3.2 The $r$-th power of $C_{n}$ packs with every graph $G$ on $n$ vertices with $\Delta(G) \leq \frac{n}{r+1}-1$.

Seymour's conjecture implies the packing version of the Hajnal-Szemerédi Theorem, since any $r+1$ consecutive vertices of the $r$-th power of a cycle induce $K_{r+1}$. Indeed, Seymour's original motivation for his conjecture was to find an understandable proof of the Hajnal-Szemerédi Theorem. Fan and Kierstead [29] came close to proving 
Posa's conjecture, but had to settle for finding the square of a hamiltonian path, albeit with a slightly weaker hypothesis.

Theorem 3.3 (Fan and Kierstead [29]) Every graph $G$ on $n$ vertices which satisfies $\delta(G) \geq \frac{2 n-1}{3}$ contains the square of $P_{n}$.

The following example shows that the theorem is best possible for paths.

Example 3.4 Let $G$ be the complete tripartite graph $K_{t-1, t+1, t+1}$. Then $n:=$ $|G|=3 t+1$ and $\delta(G)=2 t=\frac{2 n-2}{3}$. If $H$ is a square path in $G$, then any three consecutive vertices must be in distinct parts. It follows that $G$ does not contain the square of $P_{n}$, since when the last vertex in the small part is used there will still be four unused vertices. Notice also that adding edges inside the small part will not create the square of $P_{n}$.

Not only does the weakened hypothesis give the best possible result with respect to paths, but it also implies a strengthened version of the Aigner-Brandt Theorem. Observe that every graph $H$ on $n$ vertices with $\Delta(H) \leq 2$ is contained in the square of $P_{n}$. In other words, the square of $P_{n}$ is universal with respect to graphs $H$ on $n$ vertices with $\Delta(H) \leq 2$.

Theorem 3.5 (Fan and Kierstead [29]) There exists a universal graph $U$ on $n$ vertices with $\Delta(U)=4$ such that (1) $U$ packs with every graph $G$ on $n$ vertices with $\Delta(G) \leq \frac{n-2}{3}$ and (2) $U$ contains every graph $H$ on $n$ vertices with $\Delta(H) \leq 2$.

Fan and Kierstead [30] also proved:

Theorem 3.6 (Fan and Kierstead [30]) If $G$ is a graph on $n$ vertices such that $\delta(G) \geq \frac{2}{3} n$, then either (1) $G$ contains the square of $C_{n}$ or (2) $G$ does not contain the square of $C_{k}$ for $k>\frac{2}{3} n$, but there exist integers $i$ and $j$ with $i+j=n$ and $i \leq j \leq \frac{2}{3} n$ such that $G$ contains the squares of vertex disjoint copies of $C_{i}$ and $C_{j}$.

Pósa's Conjecture remains open, but Komlós, Sárkőzy and Szemerédi $[49,50]$ have used Szemerédi's Regularity Lemma and their own Blow-Up Lemma [48] to prove it, and the more general conjecture of Seymour, for huge graphs.

Theorem 3.7 (Komlós, Sárkőzy and Szemerédi [50]) For every positive integer $r$ there exists an integer $N$ such that for every $n>N$ every graph $G$ on $n$ vertices with $\delta(G) \geq \frac{r}{r+1} n$ contains the $r$-th power of a hamiltonian cycle.

It is well known that every balanced bipartite graph $G$ on $n=2 s$ vertices with $\delta(G) \geq \frac{s+1}{2}$ contains $C_{n}$. Wang [76] conjectured that if $\delta(G) \geq \frac{s}{2}+1$ then $G$ is universal with respect to bipartite graphs $H$ on $n$ vertices with $\delta(H) \leq 2$. He showed that if true, this is best possible.

Example 3.8 Let $G$ be the balanced bipartite graph on $n=2 s=4 t+2$ vertices formed by taking two copies of $K_{t, t+1}$ and joining the two larger parts by a matching with $t+1$ edges. Then $\delta(G)=t+1=\frac{s+1}{2}$, but $G$ does not contain $C_{s+1}+C_{s-1}$. 
Define the ladder $L_{n}$ to be the balanced bipartite graph on $n=2 s$ vertices $a_{1}, b_{1}, \cdots, a_{s}, b_{s}$ such that $a_{i} b_{j} \in E\left(L_{n}\right)$ if and only if $|i-j| \leq 1$. It is easy to check that $L_{n}$ is universal with respect to all bipartite graphs $H$ on $n$ vertices with $\Delta(H) \leq 2$. Czygrinow and Kierstead [22] used the Regularity and Blow-Up lemmas to prove the following strengthening of Wang's conjecture for huge graphs.

Theorem 3.9 (Czygrinow and Kierstead [22]) There exists an integer $N$ such that every balanced bipartite graph $G$ on $n=2 s>N$ vertices with $\delta(G) \geq \frac{s}{2}+1$ contains $L_{n}$.

Amar [5] posed the following version of an Ore-type conjecture.

Conjecture 3.10 (Amar [5]) Every balanced $A, B$-bigraph $G$ on $n=2 s$ vertices satisfying $d(a)+d(b) \geq s+k$ for all $a \in A$ and $b \in B$ contains a copy of every balanced bipartite graph $H$ on $n$ vertices with $k$ components and $\Delta(H) \leq 2$.

Bondy and Chvátal [13] proved the case $k=1$ (prior to the conjecture) and Amar [5] proved the case $k=2$. Recently Czygrinow, DeBiasio and Kierstead [23] proved the following two theorems. Together they imply Amar's conjecture for sufficiently large graphs. Moreover, Theorem 3.12 shows that the degree bound can be relaxed to $d(a)+d(b) \geq s+2$ as long as $n$ is large in terms of $k$.

Theorem 3.11 (Czygrinow, DeBiasio and Kierstead [23]) For some integer $K$, every balanced $A, B$-bigraph $G$ on $n=2 s$ vertices satisfying $d(a)+d(b) \geq s+K$ for all $a \in A$ and $b \in B$ contains $L_{n}$.

Theorem 3.12 (Czygrinow, DeBiasio and Kierstead [23]) For every integer $k$ there exists an integer $N_{k}$ such that every balanced $A, B$-bigraph $G$ on $n=2 s>N_{k}$ vertices satisfying $d(a)+d(b) \geq s+2$ for all $a \in A$ and $b \in B$ contains a copy of every balanced bipartite graph $H$ on $n$ vertices with $k$ components and $\Delta(H) \leq 2$. Moreover if $\delta(G) \geq \frac{1}{100 k} s$, then $G$ contains $L_{n}$.

\section{Coloring}

As mentioned in the introduction, various coloring problems are important instances of packing problems. An obvious (but sharp) Dirac-type bound on the (ordinary) chromatic number is

$$
\chi(G) \leq \Delta(G)+1
$$

where $\chi(G)$ is the chromatic number of $G$. Brooks' Theorem below characterizes the graphs for which (4.1) holds with equality.

Theorem 4.1 (Brooks) If $\chi(G)=\Delta(G)+1$, then either $G$ contains the complete graph $K_{\Delta(G)+1}$ or $\Delta(G)=2$ and $G$ contains an odd cycle.

The counterpart of (4.1) for $\theta(G)$ is

$$
\chi(G) \leq\lfloor\theta(G) / 2\rfloor+1 .
$$



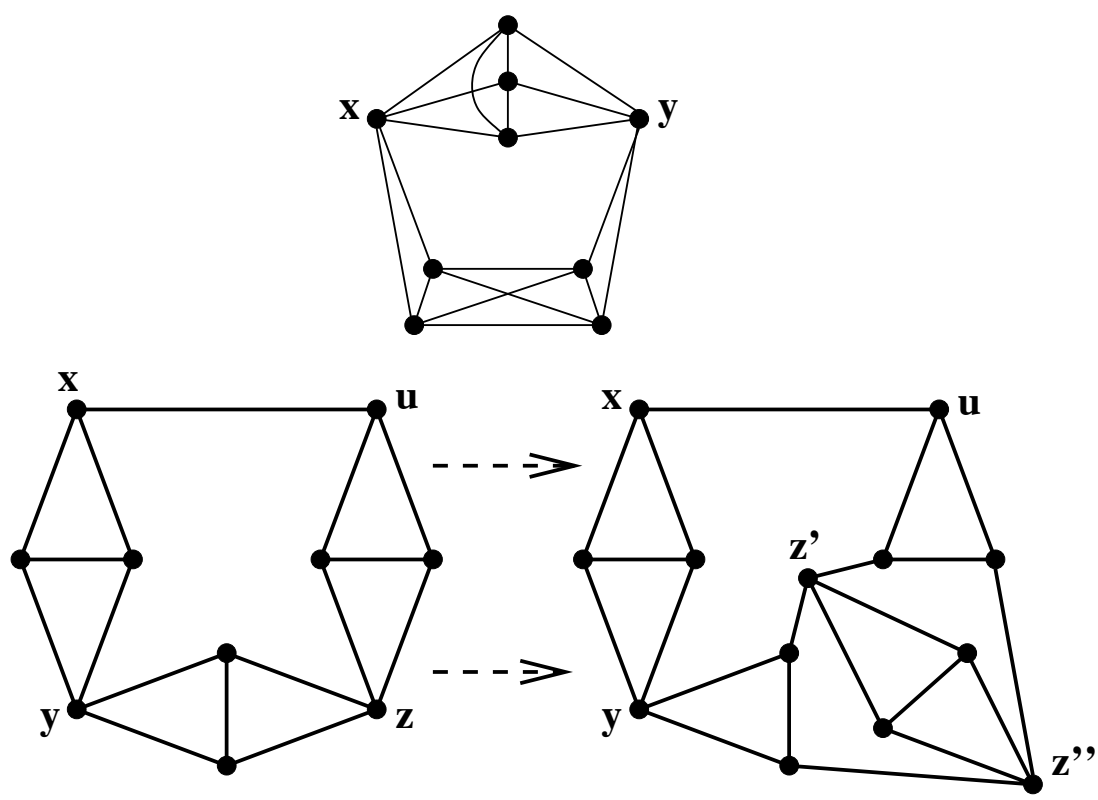

Figure 1: Above: a graph with $\theta=9$ and $\chi=5$. Below: two graphs with $\theta=7$ and $\chi=4$.

The proof is also obvious and the bound is also attained at complete graphs. However for small odd $\theta$ there are more connected graphs for which (4.2) holds with equality.

Example 4.2 Let $G$ be the top graph in Figure 1, i.e., the 9-vertex graph with $V(G)=Q \cup Q^{\prime}, Q \cap Q^{\prime}=\{x, y\}, G[Q]=K_{5}-x y$, and $G\left[Q^{\prime}\right]=K_{6}-T$, where $T$ is a tree with four leaves, $x y \in E(T)$ and $d(x)=3=d(y)$. Since $x$ and $y$ are the only vertices with degree greater than 4 and are not adjacent to each other, $\theta(G)=9$. Every (proper) 4-coloring of $G[Q]$ assigns $x$ and $y$ the same colors, since $G\left[N_{Q}(x) \cap N_{Q}(y)\right]=K_{3}$. On the other hand, every 4-coloring of $G\left[Q^{\prime}\right]$ assigns $x$ and $y$ different colors, since $G\left[N_{Q^{\prime}}(x) \cup N_{Q^{\prime}}(y)\right]=K_{4}$. Thus $\chi(G)>4$.

Example 4.3 Let $G$ be a graph with $\theta(G) \leq 7$ and $\chi(G)=4$ (for example, $G=$ $\left.K_{4}\right)$. We construct from $G$ a $(3+|V(G)|)$-vertex graph $G^{\prime}$ with $\theta\left(G^{\prime}\right) \leq 7$ and $\chi\left(G^{\prime}\right)=4$ as follows. Choose a vertex $v \in V(G)$ that has no neighbors of degree 4 (each vertex of degree 4 has this property). Split $v$ into two vertices $v_{1}$ and $v_{2}$ of degree at most two. Add two new vertices $x_{v}$ and $y_{v}$ that are adjacent to $v_{1}, v_{2}$, and to each other. By construction, $\theta\left(G^{\prime}\right)=7$. Suppose that $G^{\prime}$ has a 3-coloring $f$. Since both, $v_{1}$ and $v_{2}$ are adjacent to $x_{v}$ and $y_{v}$, we need $f\left(v_{1}\right)=f\left(v_{2}\right)$. But then $f$ yields a 3 -coloring of $G$, contrary to our assumption. The two bottom graphs in Figure 1 illustrate the idea (with $G$ on the left).

Iterating the idea of Example 4.3 yields infinitely many 2-connected graphs $G$ with $\theta(G) \leq 7$ and $\chi(G)=4$. In contrast, for graphs with Ore-degree at least 12 (i.e., with chromatic number at least 7 ), the only extremal connected graphs are complete graphs. 
Theorem 4.4 ([41]) If $7 \leq \chi(G)=\lfloor\theta(G) / 2\rfloor+1$, then $G$ contains the complete graph $K_{\chi(G)}$.

We think that the statement of Theorem 4.4 holds also for graphs $G$ with Oredegree at least 10 but cannot prove it. An even more challenging task would be to describe connected graphs $G$ with $\theta(G) \leq 7$ and $\chi(G)=4$.

\section{Equitable coloring}

In several applications of coloring as a partition problem there is an additional requirement that color classes be not too large or be of approximately the same size. Examples are the mutual exclusion scheduling problem [6, 71], scheduling in communication systems [33], construction timetables [46], and round-the-clock scheduling [74]. For other applications in scheduling, partitioning, and load balancing problems, one can look into [7, 58, 71]. A model imposing such a requirement is equitable coloring - a proper coloring such that color classes differ in size by at most one. As mentioned in the introduction, equitable coloring is a particular case of the general graph packing problem. Alon and Füredi [2], Pemmaraju [65] and Janson, Łuczak, and Ruciński [34] used equitable colorings to give new bounds on tails of distributions of sums of random variables. Rödl and Ruciński [66] used equitable colorings to give a new proof of the Blow-Up Lemma.

In contrast to ordinary coloring, a graph may have an equitable $k$-coloring (i.e., an equitable coloring with $k$ colors) but have no equitable $(k+1)$-coloring. Thus, it is natural to look for the minimum number, eq $(G)$, such that for every $k \geq \operatorname{eq}(G)$, $G$ has an equitable $k$-coloring.

Finding eq $(G)$ even for planar graphs $G$ is an $N P$-hard problem. This motivates a series of extremal problems on equitable colorings. The Dirac-type Theorem 1.7 by Hajnal and Szemerédi [31] in the original language is as follows.

Theorem 5.1 (Hajnal and Szemerédi [31]) Every graph $G$ with maximum degree at most $r$ has an equitable $(r+1)$-coloring.

The proof was long and sophisticated. A shorter proof appeared in [38]. Then Kierstead, Kostochka, Mydlarz, and Szemerédi [45] devised an algorithm that in time $O\left(r n^{2}\right)$ finds an equitable $(r+1)$-coloring for any $n$-vertex graph with maximum degree at most $r$. It is based on a modification of the proof of Theorem 5.1 in [38]. Here we present a yet shorter and simpler proof of Theorem 5.1.

Proof of Theorem 5.1. Let $G$ be a graph with $\Delta(G) \leq r$. We may assume that $|G|$ is divisible by $r+1$ : If $|G|=s(r+1)-p$, where $p \in[r]$, then set $G^{\prime}=G+K_{p}$. By construction, $\left|G^{\prime}\right|$ is divisible by $r+1$ and $\Delta\left(G^{\prime}\right) \leq r$. Moreover, the restriction of any equitable $(r+1)$-coloring of $G^{\prime}$ to $G$ is an equitable $(r+1)$-coloring of $G$. So we may assume $|G|=(r+1) s$.

We argue by induction on $\|G\|$. The base step $\|G\|=0$ is trivial, so consider the induction step. Let $u$ be a non-isolated vertex. By the induction hypothesis, there exists an equitable $(r+1)$-coloring of $G-E(u)$, where $E(u)$ denotes the set of edges incident with $u$. We are done unless some color class $V$ contains an edge $u v$. Since $\Delta(G) \leq r$, some color class $T$ contains no neighbors of $u$. Moving $u$ to $T$ yields an 
$(r+1)$-coloring of $G$ with all classes of size $s$, except for one small class $V^{-}=V-u$ of size $s-1$ and one large class $V^{+}=T+u$ of size $s+1$. Such a coloring is called nearly equitable.

Given a nearly equitable $(r+1)$-coloring, define an auxiliary digraph $\mathcal{H}$, whose vertices are the color classes, so that $U W$ is a directed edge if and only if some vertex $y \in U$ has no neighbors in $W$. In this case we say that $y$ witnesses edge $U W$. Let $\mathcal{A}$ be the set of classes from which $V^{-}$can be reached in $\mathcal{H}$ and $\mathcal{B}$ be the set of classes not in $\mathcal{A}$. Set $a=|\mathcal{A}|, b=|\mathcal{B}|, A=\bigcup \mathcal{A}$ and $B=\bigcup \mathcal{B}$. Then $r+1=a+b$. Since every vertex $y \in B$ has a neighbor in every class of $\mathcal{A}$,

$$
d_{A}(y) \geq a \text { for all } y \in B .
$$

Case 0: $V^{+} \in \mathcal{A}$. Then there exists a $V^{+}, V^{-}$-path $\mathcal{P}=V_{1}, \ldots, V_{k}$ in $\mathcal{H}$. Moving each witness $y_{j}$ of $V_{j} V_{j+1}$ to $V_{j+1}$ yields an equitable $(r+1)$-coloring of $G$.

We now argue by a secondary induction on $b$, whose base step $b=0$ holds by Case 0. If $V^{+} \notin \mathcal{A}$, then $|A|=a s-1$ and $|B|=b s+1$. Consider the secondary induction step.

A class $W \in \mathcal{A}$ is terminal if every $U \in \mathcal{A}-W$ can reach $V^{-}$in $\mathcal{H}-W$. Let $\mathcal{A}^{\prime}$ be the set of terminal classes, $a^{\prime}=\left|\mathcal{A}^{\prime}\right|$ and $A^{\prime}=\bigcup \mathcal{A}^{\prime}$. An edge $w z$ is solo if $w \in W \in \mathcal{A}^{\prime}, z \in B$ and $N_{W}(z)=\{w\}$. Ends of solo edges are solo vertices and solo neighbors of each other.

If $V^{-} \in \mathcal{A}^{\prime}$ then no other class can be in $\mathcal{A}$. Thus in this case $a=1, b=r$ and by $\left(^{*}\right),|E(A, B)| \geq|B|=r s+1$, a contradiction to the fact that $|E(A, B)| \leq r|A|=$ $r(s-1)$. So, $V^{-} \notin \mathcal{A}^{\prime}$.

Suppose that some solo vertex $w \in W \in \mathcal{A}^{\prime}$ witnesses an edge $W X \in E(\mathcal{H}[A])$. Let $y \in B$ be a solo neighbor of $w$. Move $w$ to $X$ and $y$ to $W$. This yields nearly equitable colorings of $G[A+y]$ and $G[B-y]$. Since $W$ is terminal, $X+w$ can reach $V^{-}$in $\mathcal{H}-W$. Thus by Case $0, G[A+y]$ has an equitable $a$-coloring. By $\left(^{*}\right), \Delta(G[B-y]) \leq b-1$. So by the primary induction hypothesis, $G[B-y]$ has an equitable $b$-coloring. Combining these equitable colorings yields an equitable $(r+1)$-coloring of $G$. Thus, we may assume:

Each solo vertex $z \in A^{\prime}$ witnesses no edges in $\mathcal{H}$, and so $d_{A}(z) \geq a-1$.

Order $\mathcal{A}$ as $X_{0}, X_{1}, \ldots, X_{a-1}$ so that $X_{0}=V^{-}$and each $X_{i}$ has a previous out-neighbor.

Case 1: For some $a-b \leq i \leq a-1$, class $X_{i}$ is not terminal. This includes the case $a \leq b$. Then some $X_{j} \in \mathcal{A}^{\prime}$ cannot reach $V^{-}$in $\mathcal{H}-X_{i}$. So $j>i$ and $X_{j}$ has no out-neighbors before $X_{i}$. In particular, $d_{\mathcal{A}}^{+}\left(X_{j}\right)<b$. Then for each $w \in X_{j}, d_{A}(w) \geq a-b$, and so $d_{B}(w)<2 b$. Let $S$ be the set of solo vertices in $X_{j}$, and $D=X_{j} \backslash S$. By definition, each $v \in B$ either has a neighbor in $S$ or at least two neighbors in $D$. Thus $\sum_{w \in X_{j}} d_{B}(w) \geq 2|B|-\left|N_{B}(S)\right|$. By $\left.{ }^{* *}\right)$, $\sum_{w \in S} d_{A}(w) \geq(a-1)|S|$ and hence $\left|N_{B}(S)\right| \leq b|S|$. Since $|S|+|D|=s$, we have the following contradiction:

$$
\begin{gathered}
r s \geq \sum_{w \in X_{j}}\left(d_{A}(w)+d_{B}(w)\right) \geq(a-b)|D|+(a-1)|S|+2|B|-\left|N_{B}(S)\right| \geq \\
\geq(a-b)|D|+(a-1)|S|+2 b s+2-b|S|=(a+b)|D|+2+(a+b-1)|S|>r s .
\end{gathered}
$$


Case 2: All the last $b$ classes $X_{a-b}, \ldots, X_{a-1}$ are terminal. Then $a^{\prime} \geq b$. For $y \in B$, let $\sigma(y)$ be the number of solo neighbors of $y$. For each $y \in B$,

$$
r \geq d(y) \geq a+d_{B}(y)+\left(a^{\prime}-\sigma(y)\right) \geq r+1+d_{B}(y)+a^{\prime}-b-\sigma(y) .
$$

So $\sigma(y) \geq a^{\prime}-b+d_{B}(y)+1$. Let $I$ be a maximal independent set with $V^{+} \subseteq I \subseteq B$. Then $\sum_{y \in I}\left(d_{B}(y)+1\right) \geq|B|=b s+1$. Since $a^{\prime} \geq b$,

$$
\sum_{y \in I} \sigma(y) \geq \sum_{y \in I}\left(a^{\prime}-b+d_{B}(y)+1\right) \geq s\left(a^{\prime}-b\right)+b s+1>a^{\prime} s=\left|A^{\prime}\right| .
$$

So some vertex $w \in W \in \mathcal{A}^{\prime}$ has two solo neighbors $y_{1}$ and $y_{2}$ in the independent set $I$.

By the primary induction hypothesis, we can equitably $b$-color $G\left[B-y_{1}\right]$. Let $Y^{\prime}$ be the class of $y_{2}$ in this coloring. By $\left({ }^{* *}\right), d_{B-y_{1}}(w) \leq r-(a-1)-1=b-1$ and we can move $w$ to some class $U \subseteq B-y_{1}$. Replacing $w$ with $y_{1}$ in $W$ to get $W^{*}$ and moving $w$ to $U$ yields a new nearly equitable $(r+1)$-coloring of $G$. Now at least $a+1$ classes, $W^{*}, Y^{\prime}$, and all $X \in \mathcal{A}^{\prime}-W$, can reach $V^{-}$. In this case we are done by the secondary induction hypothesis.

As discussed in Example 2.3, if $r$ is odd, then $K_{r, r}$ has no equitable $r$-coloring. Chen, Lih and $\mathrm{Wu}$ [17] proposed the following common strengthening of Theorem 5.1 and Brooks' theorem.

Conjecture 5.2 (Chen, Lih and Wu [17]) Let $G$ be a connected graph such that $\Delta(G)=r$. Then $G$ has no equitable $r$-coloring if and only if either (1) $G=K_{r+1}$, or (2) $r=2$ and $G$ is an odd cycle, or (3) $r$ is odd and $G=K_{r, r}$.

Some partial cases of Conjecture 5.2 were proved in $[17,52,62,79,80]$. In particular, Chen, Lih and $\mathrm{Wu}[17]$ proved that the conjecture holds for $r=3$ :

Theorem 5.3 (Chen, Lih and Wu [17]) Let $G$ be a connected graph such that $\Delta(G) \leq 3$. Then $G$ has no equitable 3-coloring if and only if $G=K_{4}$ or $G=K_{3,3}$.

Brooks' theorem characterizes all graphs with maximum degree $r$ that are $r$ colorable, since a graph is $r$-colorable if and only if each of its components is. This is not the case for equitable $r$-coloring. For example, for each odd $r \geq 3$, the graph consisting of two disjoint copies of $K_{r, r}$ has an equitable $r$-coloring, but the graph consisting of a copy of $K_{r, r}$ and a copy of $K_{r}$ does not. This construction can be generalized. Say that a graph $H$ is $r$-equitable if $|H|$ is divisible by $r, H$ is $r$-colorable and every $r$-coloring of $H$ is equitable. If $G$ contains $K_{r, r}$ and $G-K_{r, r}$ is $r$-equitable, then $G$ does not have an equitable $r$-coloring. This motivates the study of equitable graphs, i.e., graphs that are $r$-equitable for some $r$. It was proved in [40] that there is a good description of the family of all $r$-equitable graphs; they can all be built from simple examples in a straightforward way.

If an $r$-colorable graph $G$ has a spanning subgraph whose components are all $r$-equitable, then $G$ is also $r$-equitable. We say that an $r$-equitable graph $G$ is $r$ reducible if $V(G)$ has a partition $\left\{V_{1}, \ldots V_{t}\right\}$ into at least two parts such that $G\left[V_{i}\right]$ is $r$-equitable for each $i \in[t]$; otherwise $G$ is $r$-irreducible. Clearly $K_{r}$ is $r$-irreducible. The reader can see one other 5 -irreducible graph $F_{1}$ and three other 4 -irreducible 

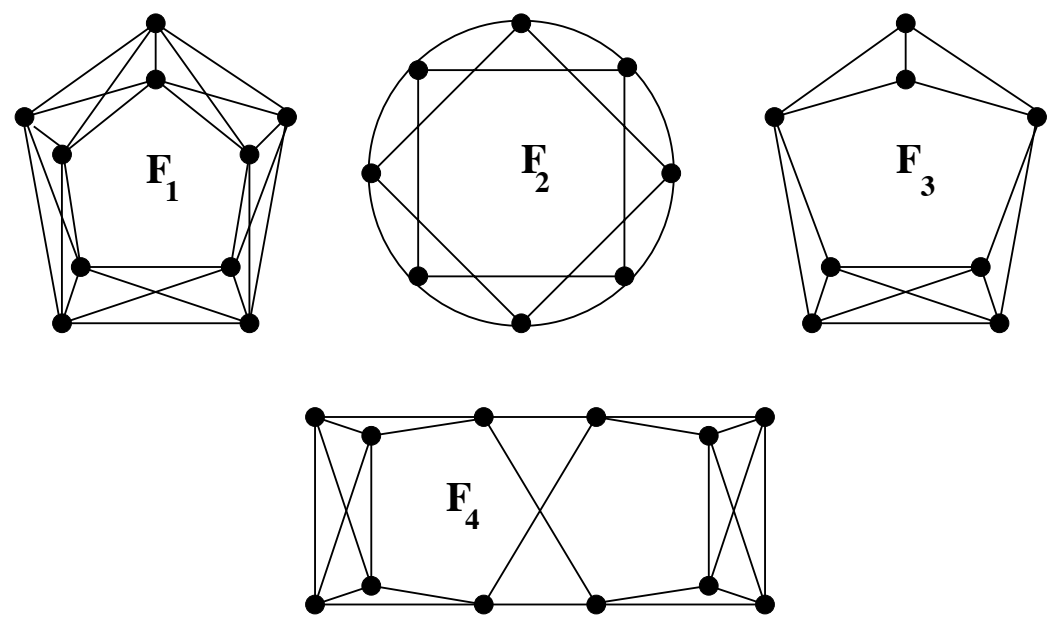

Figure 2: One 5-equitable and three 4-equitable basic graphs.
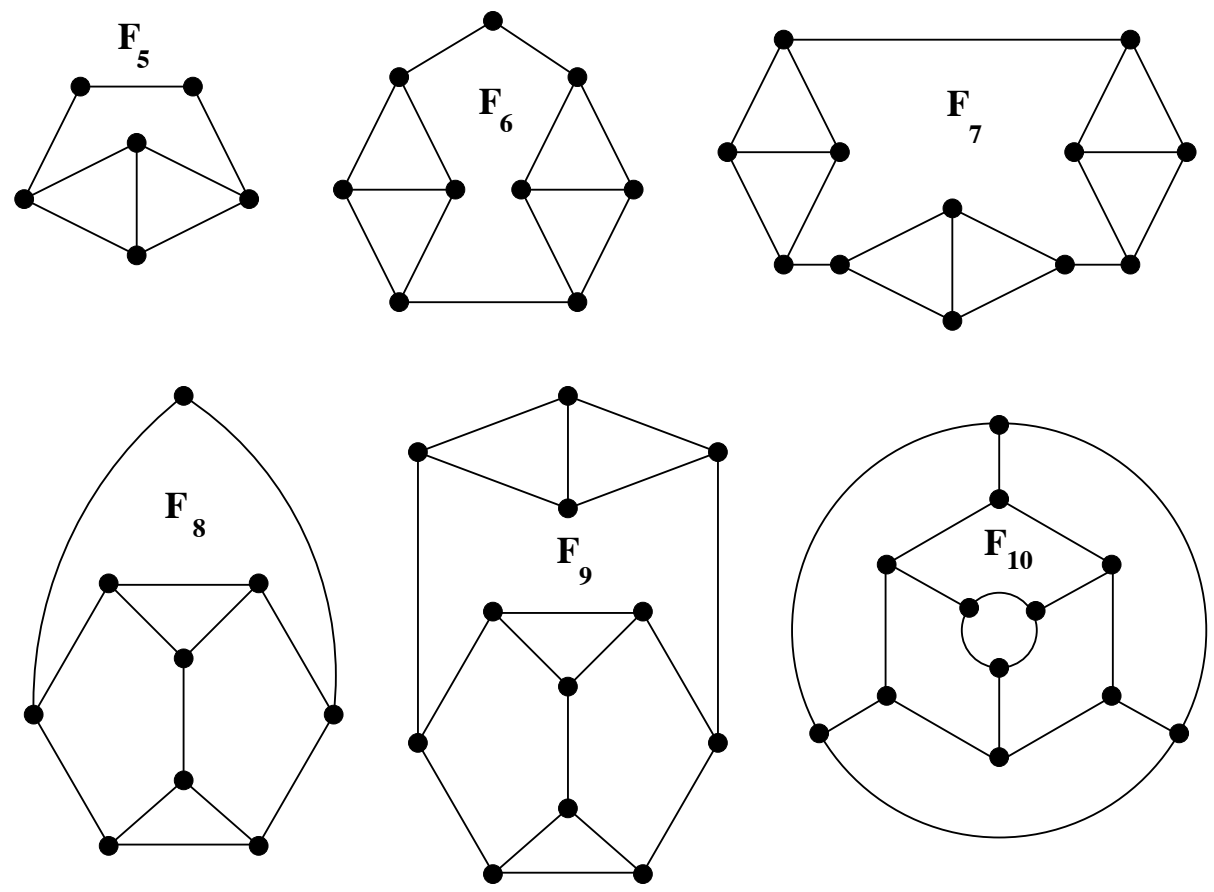

Figure 3: Six 3-equitable basic graphs. 
graphs $F_{2}, F_{3}, F_{4}$ in Figure 2 and six other 3 -irreducible graphs $F_{5}, \ldots, F_{10}$ in Figure 3. Together with $K_{r}$, the $r$-irreducible graphs from this list are the $r$-basic graphs. An $r$-decomposition of $G$ is a partition of $V(G)$ into subsets $V_{1}, \ldots, V_{t}$ such that each $G\left[V_{i}\right]$ is $r$-basic. We say that $G$ is $r$-decomposable if it has an $r$ decomposition. As was just mentioned, if $r$ is odd and $G$ is $r$-decomposable, then $G \cup K_{r, r}$ has no equitable $r$-coloring. It was conjectured in [40] that this is the only obstacle that prevents an $r$-colorable graph with $\Delta(G) \leq r$ from having an equitable $r$-coloring.

Conjecture 5.4 ([40]) Suppose that $r \geq 3$ and $G$ is an $r$-colorable graph with $\Delta(G)=r$. Then $G$ has no equitable $r$-coloring if and only if $r$ is odd and there exists $H \subseteq G$ such that $H=K_{r, r}$ and $G-H$ is $r$-decomposable.

For $r \geq 6$, this conjecture means that if an $r$-colorable graph $G$ with $\Delta(G) \leq r$ has no equitable $r$-coloring, then $r$ is odd and $V(G)$ can be partitioned into sets $V_{0}, \ldots, V_{t}$ such that $G\left[V_{0}\right]=K_{r, r}$ and $G\left[V_{i}\right]=K_{r}$ for each $i=1, \ldots, r$.

A nearly equitable $r$-coloring of a graph $G$ is defined in the above proof of Theorem 5.1. In this case $|G|$ is divisible by $r$. If $r$ is odd, $G$ contains $K_{r, r}$ and $G-K_{r, r}$ has a nearly equitable $r$-coloring, then $G$ has an equitable $r$-coloring, since the small class of one of the components can be combined with the large class of the other. This explains our interest in nearly equitable $r$-colorings.

Let $\mathcal{G}(r)$ be the class of all graphs $G$ with $\Delta(G) \leq r$ and $\chi(G) \leq r$. Let $\mathcal{G}(r, n)$ be the set of graphs in $\mathcal{G}(r)$ with at most $n$ vertices. The following result implies that Conjectures 5.4 and 5.2 are equivalent.

Theorem 5.5 ([40]) Let $G \in \mathcal{G}(r)$ with $|G|$ divisible by $r$. The following are equivalent:

(A) $G$ is r-decomposable;

(B) $G$ is r-equitable;

(C) $G$ has an equitable $r$-coloring, but does not have a nearly equitable $r$-coloring.

Corollary 5.6 ([40]) For all positive integers $r$ and $n>r$, Conjecture 5.2 holds for all graphs in $\mathcal{G}(r, n)$ if and only if Conjecture 5.4 holds for all graphs in $\mathcal{G}(r, n)$.

Corollary 5.7 ([40]) Let $G \in \mathcal{G}(r)$ be $r$-equitable. Then $G$ has a unique $r$-decomposition.

Corollary 5.8 ([40]) There exists a polynomial time algorithm for deciding if a graph $G \in \mathcal{G}(r)$ is r-equitable.

Very recently, the case $r=4$ of Conjecture 5.2 was proved [42].

The following Ore-type analogue of Theorem 5.1 (slightly strengthening a conjecture in $[54,56])$ was proved in [39].

Theorem 5.9 ([39]) For every $r \geq 3$, each graph $G$ with $\theta(G) \leq 2 r+1$ has an equitable $(r+1)$-coloring. 
In particular, the theorem yields that Conjecture 5.2 holds for graphs in which vertices of maximum degree form an independent set. The bounding examples for this theorem (apart from $K_{r+1}$ ) are graphs $K_{m, 2 r-m}$ for every odd $0<m \leq r$. In the same paper, the following Ore-type analogue of Conjecture 5.2 was proposed.

Conjecture 5.10 ([39]) Let $r \geq 3$ and $G$ be a connected graph with $\theta(G) \leq 2 r$. If $G$ is distinct from $K_{r+1}$ and from $K_{m, 2 r-m}$ for odd $m$, then $G$ has an equitable $r$-coloring.

Conjecture 5.10 was proved to hold for $r=3$. In [41] the following common extension of Conjectures 5.4 and 5.10 was posed.

Conjecture 5.11 ([41]) Suppose that $r \geq 3$. An $r$-colorable, $n$-vertex graph $G$ with $\theta(G) \leq 2 r$ has no equitable $r$-coloring, if and only if $n$ is divisible by $r$, and there exists $W \subseteq G$ such that $W=K_{m, 2 r-m}$ for some odd $m$ and $G-W$ is $r$-decomposable.

It was proved in [41] that Conjecture 5.11 is equivalent to Conjecture 5.10 even for graphs with restricted number of vertices and restricted values of Ore-degree.

Theorem 5.12 ([41]) Assume that Conjecture 5.10 holds for all graphs with at most $n$ vertices and Ore-degree at most $2 r$. Let $G$ be an $r$-colorable $n$-vertex graph with $\theta(G) \leq 2 r$. Then $G$ has no equitable $r$-coloring if and only if $n$ is divisible by $r$ and there exists $H \subseteq G$ such that $H=K_{m, 2 r-m}$ for some odd $m$ and $G-H$ is $r$-decomposable.

It follows that Conjecture 5.11 holds for $r=3$.

\section{Equitable list coloring}

A list analogue of equitable coloring was introduced by Kostochka, Pelsmajer and West [53]. A list assignment $L$ for a graph $G$ assigns to each vertex $v \in V(G)$ a set $L(v)$ of allowable colors. An $L$-coloring of $G$ is a proper vertex coloring such that for every $v \in V(G)$ the color on $v$ belongs to $L(v)$. Given a $k$-uniform list assignment $L$ for an $n$-vertex graph $G$, the graph $G$ is equitably $L$-colorable if $G$ has an $L$-coloring where no color is used on more than $\lceil n / k\rceil$ vertices. A graph $G$ is equitably $k$-choosable if $G$ is equitably $L$-colorable whenever $L$ is a $k$-uniform list assignment for $G$.

Because one cannot ensure the appearance of each color, the techniques previously used for ordinary equitable colorings do not work well for equitable list colorings. Nevertheless, Kostochka, Pelsmajer and West [53] suggested that the following analogue of the Hajnal-Szemerédi Theorem holds.

Conjecture 6.1 (Kostochka, Pelsmajer and West [53]) Every graph $G$ is equitably $(1+\Delta(G))$-choosable.

Furthermore, Pelsmajer [64] and independently Lih and Wang [61] confirmed this conjecture for graphs with maximum degree at most 3 :

Theorem 6.2 (Pelsmajer [64], Lih and Wang [61]) If $\Delta(G) \leq 3$, then $G$ is equitably 4-choosable. 
Very recently, the conjecture was proved for graphs with maximum degree 4 [43]. We believe that the following Ore-type analogue of Conjecture 6.1 also holds.

Conjecture 6.3 Every graph $G$ is equitably $(1+0.5 \theta(G))$-choosable.

The result below confirms the conjecture for graphs with small $\theta$ and somewhat extends Theorem 6.2.

Theorem 6.4 If $\theta(G) \leq 6$, then $G$ is equitably 4 -choosable.

Proof Let $G$ be an edge-minimal counterexample to the theorem. In particular, $|L(x)|=4$ for every $x \in V(G)$. Let $v$ be a vertex of maximum degree in $G$ and $w_{1}, \ldots, w_{d(v)}$ be the neighbors of $v$. Since $\theta(G) \leq 6, d(v) \leq 5$. If $d(v) \leq 3$, then we are done by Theorem 6.2. So, $d(v) \in\{4,5\}$.

If $d(v)=5$, then the component of $G$ containing $v$ is $K_{5,1}$. Let $G_{1}=G-v-w_{1}-$ $w_{2}-w_{3}$. Since $G_{1}$ is a proper subgraph of $G, \theta\left(G_{1}\right) \leq 6$. By the minimality of $G$, the subgraph $G_{1}$ admits some equitable $L$-coloring $f$. This means that each color is used on at most $\left\lceil\left|V\left(G_{1}\right)\right| / 4\right\rceil=\lceil|V(G)| / 4\rceil-1$ vertices. We extend $f$ to an equitable $L$-coloring of $G$ as follows: Choose a color $\alpha_{0} \in L(v)-f\left(w_{4}\right)-f\left(w_{5}\right)$ as $f(v)$, and then for $i=1,2,3$ choose a color $\alpha_{i} \in L\left(w_{i}\right)-f(v)-\left\{f\left(w_{j}\right): 1 \leq j \leq i-1\right\}$ as $f\left(w_{i}\right)$. Since each color appears on our four "new" vertices only once, the resulting coloring is equitable.

Suppose now that $d(v)=4$. Since $\theta(G) \leq 6, d\left(w_{i}\right) \leq 2$ for each $i=1,2,3,4$. For $i=1,2,3,4$, let $u_{i}$ be the neighbor of $w_{i}$ distinct from $v$, if it exists.

Case 1: $u_{1}$ does not exist or $u_{1}=w_{2}$. Consider $G_{1}=G-v-w_{1}-w_{2}-w_{3}$. By the minimality of $G$, there exists an equitable $L$-coloring $f$ of $G_{1}$. Extend $f$ to an $L$-coloring of $G$ as follows: Choose a color $\alpha \in L(v)-f\left(w_{4}\right)$ as $f(v)$, then choose $f\left(w_{3}\right) \in L\left(w_{3}\right)-f(v)-f\left(u_{3}\right), f\left(w_{2}\right) \in L\left(w_{2}\right)-f(v)-f\left(w_{3}\right)-f\left(u_{2}\right)$, and finally $f\left(w_{1}\right) \in L\left(w_{1}\right)-f(v)-f\left(w_{3}\right)-f\left(w_{2}\right)$. Again, since each color appears on our four "new" vertices only once, the resulting coloring is equitable.

So, in what follows, all $u_{i}$ exist and are distinct from all $w_{j}$.

Case 2: $\left|L(v)-L\left(w_{1}\right)\right| \geq 2$. Consider $G_{1}=G-v-w_{1}-w_{2}-w_{3}$ as in Case 1 . Let $f$ be an equitable $L$-coloring of $G_{1}$. Choose $f(v) \in L(v)-L\left(w_{1}\right)-f\left(w_{4}\right)$, then $f\left(w_{3}\right) \in L\left(w_{3}\right)-f(v)-f\left(u_{3}\right), f\left(w_{2}\right) \in L\left(w_{2}\right)-f(v)-f\left(w_{3}\right)-f\left(u_{2}\right)$, and finally $f\left(w_{1}\right) \in L\left(w_{1}\right)-f\left(u_{1}\right)-f\left(w_{3}\right)-f\left(w_{2}\right)$. Since $f(v) \notin L\left(w_{1}\right)$, the resulting coloring is proper.

From now on, $\left|L(v) \cap L\left(w_{i}\right)\right| \geq 3$ for all $i \in\{1,2,3,4\}$.

Case 3: $\left|L(v) \cap L\left(w_{1}\right) \cap L\left(w_{2}\right)\right| \geq 3$. We may assume that

$$
\left|L(v) \cap L\left(w_{1}\right)\right| \geq\left|L(v) \cap L\left(w_{2}\right)\right| .
$$

Let $G_{0}$ be obtained from $G-v-w_{3}-w_{4}$ by identifying $w_{1}$ and $w_{2}$ into a new vertex $w^{*}$. Let $L\left(w^{*}\right)=L\left(w_{2}\right)$. By the minimality of $G$, the new graph $G_{0}$ with the new list $L$ has an equitable $L$-coloring $f$.

Subcase 3.1: $f\left(w^{*}\right) \in L\left(w_{1}\right)$. We define $f\left(w_{1}\right)=f\left(w_{2}\right)=f\left(w^{*}\right)$ and let $f\left(w_{3}\right) \in$ $L\left(w_{3}\right)-f\left(u_{3}\right)-f\left(w^{*}\right), f\left(w_{4}\right) \in L\left(w_{4}\right)-f\left(u_{4}\right)-f\left(w^{*}\right)-f\left(w_{3}\right)$, and $f(v) \in L(v)-$ $f\left(w_{3}\right)-f\left(w_{4}\right)-f\left(w^{*}\right)$. 
Subcase 3.2: $f\left(w^{*}\right) \notin L\left(w_{1}\right)$. Under the conditions of Case 3, this means that $\left|L\left(w_{1}\right) \cap L\left(w_{2}\right)\right|=\left|L(v) \cap L\left(w_{1}\right) \cap L\left(w_{2}\right)\right|=3$ and now (6.1) yields that $f(w *) \notin$ $L(v)$. So, we can let $f\left(w_{2}\right)=f(w *), f\left(w_{3}\right) \in L\left(w_{3}\right)-f\left(u_{3}\right)-f\left(w^{*}\right), f\left(w_{4}\right) \in$ $L\left(w_{4}\right)-f\left(u_{4}\right)-f\left(w^{*}\right)-f\left(w_{3}\right), f\left(w_{1}\right) \in L\left(w_{1}\right)-f\left(u_{1}\right)-f\left(w_{3}\right)-f\left(w_{4}\right)$, and $f(v) \in$ $L(v)-f\left(w_{1}\right)-f\left(w_{3}\right)-f\left(w_{4}\right)$. This finishes Case 3 .

If none of Cases 1-3 takes place, then we may assume that $L(v)=\{1,2,3,4\}$ and for $i=1,2,3,4, L\left(w_{i}\right) \cap L(v)=L(v)-i$. Construct the graph $G_{0}$ with the list $L$ as in Case 3. Let $f$ be an equitable $L$-coloring of $G_{0}$. If $f\left(w^{*}\right) \neq 1$, then we extend $f$ to the whole of $G$ as in Case 3. So, assume that $f\left(w^{*}\right)=1$. If $f\left(u_{3}\right) \neq 1$, then let $f\left(w_{3}\right)=f\left(w_{2}\right)=1, f\left(w_{4}\right) \in L\left(w_{4}\right)-f\left(u_{4}\right)-1, f\left(w_{1}\right) \in L\left(w_{1}\right)-f\left(u_{1}\right)-f\left(w_{4}\right)$, and $f(v) \in\{2,3,4\}-f\left(w_{4}\right)-f\left(w_{1}\right)$. Finally, suppose $f\left(u_{3}\right)=1$. In this case, let $f\left(w_{2}\right)=1, f\left(w_{3}\right) \in L\left(w_{3}\right)-L(v), f\left(w_{4}\right) \in L\left(w_{4}\right)-f\left(u_{4}\right)-1-f\left(w_{3}\right), f\left(w_{1}\right) \in$ $L\left(w_{1}\right)-f\left(u_{1}\right)-f\left(w_{3}\right)-f\left(w_{4}\right)$, and $f(v) \in\{2,3,4\}-f\left(w_{4}\right)-f\left(w_{1}\right)$.

\section{Acknowledgements}

Research of the first author is supported in part by NSA grant H98230-08-1-0069. Research of the second author is supported in part by NSF grant DMS-06-50784 and by grant 06-01-00694 of the Russian Foundation for Basic Research. Research of the third author is partially supported by NSF grant DMS-0852452.

\section{References}

[1] M. Aigner \& S. Brandt, Embedding arbitrary graphs of maximum degree two, J. Lond. Math. Soc. 48 (1993), 39-51.

[2] N. Alon \& E. Fischer, 2-factors in dense graphs, Discrete Math. 152 (1996), $13-23$.

[3] N. Alon \& Z. Füredi, Spanning subgraphs of random graphs, Graphs. Combin. 8 (1992), 91-94.

[4] N. Alon \& J.H. Spencer, The Probabilistic Method, Second edition, Wiley, New York (2000).

[5] D. Amar, Partition of a hamiltonian graph into two cycles, Discrete Math. 58 (1986), 1-10.

[6] B. Baker \& E. Coffman, Mutual exclusion scheduling, Theor. Comput. Sci. 162 (1996), 225-243.

[7] J. Blazewicz, K. Ecker, E. Pesch, G. Schmidt \& J. Weglarz, Scheduling computer and manufacturing processes, Springer, Berlin (2001).

[8] B. Bollobás, Extremal graph theory, Academic Press, London-New York (1978).

[9] B. Bollobás \& S. E. Eldridge, Maximal matchings in graphs with given maximal and minimal degrees, Congr. Numer. XV (1976), 165-168.

[10] B. Bollobás \& S. E. Eldridge, Packing of graphs and applications to computational complexity, J. Combin. Theory Ser. B 25 (1978), 105-124. 
[11] B. Bollobás, A. V. Kostochka \& K. Nakprasit, On two conjectures on packing of graphs, Combin. Probab. Comput. 14 (2005), 723-736.

[12] B. Bollobás, A. V. Kostochka \& K. Nakprasit, Packing $d$-degenerate graphs, $J$. Combin. Theory Ser. B 98 (2008), 85-94.

[13] J. A. Bondy \& V. Chvátal, A method in graph theory, Discrete Math. 15 (1976), $111-135$.

[14] S. Brandt, An extremal result for subgraphs with few edges, J. Combin. Theory Ser. B 64 (1995), 288-299.

[15] P. A. Catlin, Subgraphs of graphs I, Discrete Math. 10 (1974), 225-233.

[16] P. A. Catlin, Embedding subgraphs and coloring graphs under extremal degree conditions, Ph.D. Thesis, Ohio State Univ., Columbus (1976).

[17] B.-L. Chen, K.-W. Lih \& P.-L. Wu, Equitable coloring and the maximum degree, European J. Combin. 15 (1994), 443-447.

[18] K. Corrádi \& A. Hajnal, On the maximum number of independent circuits in a graph, Acta Math. Acad. Sci. Hung. 14 (1963), 423-439.

[19] B. Csaba, Approximating the Bollobás-Eldridge-Catlin conjecture for bounded degree graphs, submitted.

[20] B. Csaba, On the Bollobás-Eldridge conjecture for bipartite graphs, Combin. Probab. Comput. 16 (2007), 661-691.

[21] B. Csaba, A. Shokoufandeh \& E. Szemerédi, Proof of a conjecture of Bollobás and Eldridge for graphs of maximum degree three, Combinatorica 23(1) (2003), $35-72$.

[22] A. Czygrinow \& H. A. Kierstead, 2-factors in dense bipartite graphs, Discrete Math. 257 (2002), 357-369.

[23] A. Czygrinow, L. Debiasio \& H. A. Kierstead, On 2-factors in bipartite graphs, submitted.

[24] G. Dirac, Some theorems on abstract graphs, Proc. Lond. Math. Soc. 2 (1952), 69-81.

[25] N. Eaton, A near packing of two graphs, J. Combin. Theory Ser. B 80 (2000), 98-103.

[26] H. Enomoto, On the existence of disjoint cycles in a graph, Combinatorica 18 (1998), 487-492.

[27] P. Erdős, Problem 9, in Theory of Graphs and Its Applications (ed. M. Fieldler), Czech. Acad. Sci. Publ., Prague (1964), p.159.

[28] G. Fan \& H. A. Kierstead, The square of paths and cycles, J. Combin. Theory Ser. B 63 (1995), 55-64. 
[29] G. Fan \& H. A. Kierstead, Hamiltonian square-paths, J. Combin. Theory Ser. B 67 (1996), 167-182.

[30] G. Fan \& H. A. Kierstead, Partitioning a graph into two square-cycles, J. Graph Theory 23 (1996), 241-256.

[31] A. Hajnal \& E. Szemerédi, Proof of a conjecture of Erdős, in Combinatorial Theory and its Applications (eds. P. Erdős, A. Rényi \& V. T. Sós), Vol. II, North-Holland, New York-London (1970), pp. 601-603.

[32] T. R. Jensen \& B. Toft, Graph coloring problems, Wiley-Interscience, New York (1995).

[33] S. Irani \& V. Leung, Scheduling with conflicts, and applications to traffic signal control, in Proceedings of the 7th annual ACM-SIAM symposium on discrete algorithms held in Atlanta, GA, 1996, SIAM, Philadelphia, PA (1996), pp. 8594.

[34] S. Janson, T. Łuczak \& A. Ruciński, Random Graphs, Wiley-Interscience, New York (2000).

[35] H. Kaul \& A. V. Kostochka, Extremal graphs for a graph packing theorem of Sauer and Spencer, Combin. Probab. Comput. 16 (2007), 409-416.

[36] H. Kaul, A. V. Kostochka \& G. Yu, On a graph packing conjecture by Bollobás, Eldridge, and Catlin, Combinatorica 28 (2008), 469-485.

[37] H. Kheddouci, S. Marshall, J.-F. Sacle \& M. Wozniak, On the packing of three graphs, Discrete Math. 236 (2001), 197-225.

[38] H. A. Kierstead \& A. V. Kostochka, A short proof of the Hajnal-Szemerédi Theorem on equitable coloring, Combin. Probab. Comput. 17 (2008), 265-270.

[39] H. A. Kierstead \& A. V. Kostochka, An Ore-type theorem on equitable coloring, J. Combin. Theory Ser. B 98 (2008), 226-234.

[40] H. A. Kierstead \& A. V. Kostochka, Equitable versus nearly equitable coloring and the Chen-Lih-Wu Conjecture, Combinatorica, in press.

[41] H. A. Kierstead \& A. V. Kostochka, Ore-type versions of Brooks' theorem, J. Combin. Theory Ser. B 99 (2009), 298-305.

[42] H. A. Kierstead \& A. V. Kostochka, The Chen-Lih-Wu Conjecture holds for $r=4$, in preparation.

[43] H. A. Kierstead \& A. V. Kostochka, Graphs with maximum degree 4 are equitably 5 -choosable, in preparation.

[44] H. A. Kierstead \& A. V. Kostochka, Efficient graph packing via game coloring, Combin. Probab. Comput., in press.

[45] H. A. Kierstead, A. V. Kostochka, M. Mydlarz \& E. Szemerédi, A fast algorithm for equitable coloring, Combinatorica, in press. 
[46] F. Kitagawa \& H. Ikeda, An existential problem of a weight-controlled subset and its application to school timetable construction, Discrete Math. 72 (1988), $195-211$.

[47] J. Komlós, Tiling Turán theorems, Combinatorica 20 (2000), 203-218.

[48] J. Komlós, G. Sárkőzy \& E. Szemerédi, Blow-Up Lemma, Combinatorica 17 (1997), 109-123.

[49] J. Komlós, G. Sárkőzy \& E. Szemerédi, On the Pósa-Seymour conjecture, J. Graph Theory 29 (1998), 167-176.

[50] J. Komlós, G. Sárkőzy \& E. Szemerédi, Proof of the Seymour's conjecture for large graphs, Ann. Comb. 1 (1998), 43-60.

[51] J. Komlós, G. Sárkőzy \& E. Szemerédi, Spanning trees in dense graphs, Combin. Probab. Comput. 10 (2001), 397-416.

[52] A. V. Kostochka \& K. Nakprasit, On equitable $\Delta$-coloring of graphs with low average degree, Theoret. Comput. Sci. 349 (2005), 82-91.

[53] A. V. Kostochka, M. J. Pelsmajer \& D. B. West, A list analogue of equitable coloring, J. Graph Theory 44 (2003), 166-177.

[54] A. V. Kostochka \& G. Yu, Extremal problems on packing of graphs, in Oberwolfach reports, 1, (2006), pp. 55-57.

[55] A. V. Kostochka \& G. Yu, An Ore-type analogue of the Sauer-Spencer Theorem, Graphs Combin. 23 (2007), 419-424.

[56] A. V. Kostochka \& G. Yu, Ore-type graph packing problems, Combin. Probab. Comput. 16 (2007), 167-169.

[57] A. V. Kostochka \& G. Yu, Ore-type conditions implying 2-factors consisting of short cycles, Discrete Math., in press.

[58] J. Krarup \& D. de Werra, Chromatic optimisation: Limitations, objectives, uses, references, European J. Oper. Res. 11 (1982), 1-19.

[59] D. Kühn, D. Osthus \& A. Treglown, An Ore-type theorem for perfect packings in graphs, submitted.

[60] K.-W. Lih, The equitable coloring of graphs, in Handbook of Combinatorial Optimization (eds. D.-Z. Du \& P. Pardalos), Vol. 3, Kluwer, Dordrecht (1998), pp. 543-566.

[61] K.-W. Lih \& W.-F. Wang, Equitable list coloring of graphs, Taiwanese J. Math. 8 (2004), 747-759.

[62] K.-W. Lih \& P.-L. Wu, On equitable coloring of bipartite graphs, Discrete Math. 151 (1996), 155-160.

[63] O. Ore, Note on Hamilton circuits, Amer. Math. Monthly 67 (1960), 55. 
[64] M. Pelsmajer, Equitable list-coloring for graphs of maximum degree 3, J. Graph Theory 47 (2004), 1-8.

[65] S. V. Pemmaraju, Equitable coloring extends Chernoff-Hoeffding bounds, in Approximation, randomization and combinatorial optimization (Berkeley, CA, 2001), Springer, Berlin (2001), 285-296.

[66] V. Rödl \& A. Ruciński, Perfect matchings in $\epsilon$-regular graphs and the Blow-Up Lemma, Combinatorica 19 (1999), 437-452.

[67] J.-F. Sacle \& M. Wozniak, A note on packing of three forests, Discrete Math. 164 (1997), 265-274.

[68] N. Sauer \& J. Spencer, Edge disjoint placement of graphs, J. Combin. Theory Ser. B 25 (1978), 295-302.

[69] N. Sauer \& H. Wang, Packing of three copies of a graph, J. Graph Theory 21 (1996), 71-80.

[70] P. Seymour, Problem section, in Combinatorics: Proceedings of the British Combinatorial Conference, 1973 (eds. T. P. McDonough \& V. C. Mavron), Cambridge University Press, Cambridge (1974), pp. 201-202.

[71] B. F. Smith, P. E. Bjorstad \& W. D. Gropp, Domain decomposition. Parallel multilevel methods for elliptic partial differential equations, Cambridge University Press, Cambridge (1996).

[72] S. Teo \& H. P. Yap, Packing two graphs of order $n$ having total size at most $2 n-2$, Graphs Combin. 6 (1990), 197-205.

[73] S. Teo \& H. P. Yap, Two theorems on packings of graphs, European J. Combin. 8 (1987), 199-207.

[74] A. Tucker, Perfect graphs and an application to optimizing municipal services, SIAM Review 15 (1973), 585-590.

[75] H. Wang, On the maximum number of independent cycles in a graph, Discrete Math. 205 (1999), 183-190.

[76] H. Wang, On 2-factors of a bipartite graph, J. Graph Theory 31 (1999), 101106.

[77] M. Wozniak, Packing of graphs, Dissertationes Math. (Rozprawy Mat.) 362 (1997), 1-78.

[78] H. P. Yap, Packing of graphs — a survey, Discrete Math. 72 (1988), 395-404.

[79] H. P. Yap \& Y. Zhang, The equitable $\Delta$-colouring conjecture holds for outerplanar graphs, Bull. Inst. Math. Acad. Sin. 25 (1997), 143-149.

[80] H.-P. Yap \& Y. Zhang, Equitable colourings of planar graphs, J. Combin. Math. Combin. Comp. 27 (1998), 97-105. 
[81] X. Zhu, Refined activation strategy for the marking game, J. Combin. Theory Ser. B 98 (2008), 1-18.

Department of Mathematics and Statistics, Arizona State University, Tempe, AZ 85287, USA kierstead@asu.edu

Department of Mathematics, University of Illinois, Urbana, IL, 61801, USA and Sobolev Institute of Mathematics, Novosibirsk, 630090, Russia kostochk@math.uiuc.edu

Department of Mathematics, College of William and Mary Williamsburg, VA 23187, USA gyu@wm.edu 